\title{
Editorial. Dialogues sur la violence et la démocratie en France et au Brésil
}

Angelina Peralva et Sérgio Adorno

\section{(2) OpenEdition}

\section{Journals}

Édition électronique

URL : http://journals.openedition.org/conflits/1873

DOI : $10.4000 /$ conflits.1873

ISSN : $1777-5345$

Éditeur :

CCLS - Centre d'études sur les conflits lilberté et sécurité, L'Harmattan

Édition imprimée

Date de publication : 1 septembre 2005

Pagination : 5-9

ISBN : 2-7475-9476-9

ISSN : 1157-996X

Référence électronique

Angelina Peralva et Sérgio Adorno, «Editorial. Dialogues sur la violence et la démocratie en France et au Brésil », Cultures \& Conflits [En ligne], 59 | automne 2005, mis en ligne le 09 février 2006, consulté le 30 mars 2021. URL : http://journals.openedition.org/conflits/1873 ; DOI : https://doi.org/10.4000/ conflits. 1873

Ce document a été généré automatiquement le 30 mars 2021.

Creative Commons License 


\title{
Editorial. Dialogues sur la violence et la démocratie en France et au Brésil
}

\author{
Angelina Peralva et Sérgio Adorno
}

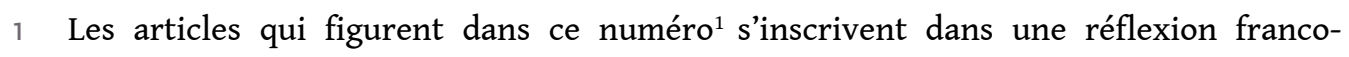
brésilienne, développée dans le cadre des accords de coopération CAPES-COFECUB et USP-COFECUB, liant depuis plusieurs années des chercheurs du Centre d'Analyse et d'Intervention Sociologiques (CADIS-EHESS/CNRS) et du Núcleo de Estudos da Violência da Universidade de São Paulo (NEV-USP). Nous remercions la rédaction de Cultures et Conflits d'avoir accepté d'ouvrir les pages de la revue à une publication partielle de notre travail.

2 Nos échanges ont eu comme point de départ la constatation de la semblable importance que revêtait, au Brésil et en France, le débat sur la violence - alors que les faits de violence eux-mêmes n'y étaient pas de même nature. Le sens de notre travail commun a d'emblée reposé sur un effort d'élucidation de cette similitude paradoxale. Côté Brésil, la violence apparaissait tout d'abord comme une quantité mesurable : 45334 homicides enregistrés en 2000 pour une population de 170 millions d'habitants, un des taux $(26 / 100000)^{2}$ les plus élevés au monde, alors qu'en France cet indicateur était parmi les plus faibles de la planète. Même si les taux de suicides sont particulièrement élevés dans l'Hexagone (23/100 000 habitants) ${ }^{3}$, il est rare qu'ils soient directement pris en compte dans les représentations de la violence - et en tout cas ils ne le sont jamais au même titre que les «incivilités urbaines » et autres marques d'agressivité dans les relations interpersonnelles, qui semblent tant inquiéter l'opinion publique française.

Nous nous trouvions donc, d'une part, face à une violence meurtrière qu'une démocratie émergente telle la brésilienne ne parvenait pas à juguler; d'autre part, face à des violences apparemment banales, mais tout de même porteuses d'une grande force de résonance médiatico-politique, alors que d'autres, bien plus graves, étaient passées sous silence. Un tel écart au niveau des faits n'a pas rendu impossible notre réflexion commune; mais il a exigé d'aller au-delà d'une perception de la violence comme un 
allant de soi, en induisant notamment une décentration par rapport aux pratiques violentes objectivables: car, soit nous renoncions à prendre au sérieux l'existence même de la "violence » en France; soit il fallait admettre de l'envisager - mais alors pour les deux pays - comme un phénomène complexe, engageant simultanément des faits et des représentations, sorte de produit finalisé d'un travail sur soi effectué par chaque collectivité nationale à partir d'une culture qui lui était propre. Autrement dit, il fallait envisager « la violence » (quelles que soient les pratiques que ce mot recouvre) comme un puissant révélateur des conditions de fonctionnement de la démocratie. C'est effectivement dans cette dernière perspective que nous avons entrepris la réflexion que voici.

4 Une grande importance a été ainsi accordée aux mythes nationaux fondateurs de la démocratie, qui dans les deux pays sont censés assurer l'égalité des citoyens. La République, qui est en France, comme on sait, bien plus qu'un simple régime politique, place la responsabilité de l'intégration nationale directement entre les mains de l'Etat. $\mathrm{Au}$ Brésil, où les limites de l'entreprise étatique républicaine ont été très tôt révélées par la répression sanglante à l'insurrection paysanne de Canudos (1897, 20000 morts), l'intégration nationale mobilise davantage et plus facilement des cadres culturels, comme il en ressort du mythe de la démocratie raciale. Malgré cette différence, de taille, les deux modèles restent proches, nous dit Alexandra Poli, en ce qu'ils produisent tous deux des effets comparables d'occultation des inégalités des "races", et plus encore du racisme. Et si cette occultation devient aujourd'hui visible, c'est grâce à la vitalité des affirmations identitaires - ethniques, culturelles, religieuses - observables au Brésil comme en France, qui donnent une nouvelle épaisseur à la question du racisme. A travers l'analyse des grandes étapes de l'action antiraciste dans les deux pays, qui passe de la condamnation générale à la prise en compte effective des expériences vécues, son article éclaire les processus de construction et de déconstruction du tabou qui entoure la reconnaissance du racisme, façonné par les mythes démocratiques qui ont fondé ces deux sociétés.

Dans les deux pays également, le journalisme et ses évolutions sont au cœur des tensions à l'oeuvre dans les représentations sur la violence. Cette thématique, autrefois du ressort exclusif de la presse populaire, a fait une entrée en force dans le débat médiatique en même temps que le journalisme subissait l'impact d'une double évolution : celle issue de l'élargissement croissant du public touché par les médias de masse, avec le brouillage corrélatif des attentes dont il serait porteur; et celle liée à l'opacité des enjeux proprement sociaux du débat. Au Brésil, la logique " grand public » qui oriente le journalisme actuel est ancienne pour la télévision, plus récente pour la presse écrite, avec une autonomie relative plus forte qu'en France vis-à-vis de la sphère politique. Ceci se traduit par une relative indépendance des journalistes de presse écrite dans le traitement des violences urbaines, tandis que la mise en scène télévisuelle de ces violences obéit à une pure logique spectaculaire/populaire. En France, cette logique " grand public » est beaucoup plus récente, autant dans la presse écrite qu'à la télévision. En revanche, l'autonomie des journalistes vis-à-vis du politique est très faible, de telle sorte que l'agenda médiatique accompagne au plus près les rhétoriques politiques dominantes à chaque moment. Eric Macé et Angelina Peralva analysent ces questions à partir de recherches menées auprès de journaux et journalistes de presse écrite et télévision, en France et au Brésil. 
Une interrogation non moins centrale concerne la manière par laquelle l'Etat gère la vie collective et répond aux diverses "violences» que celle-ci engendre. Pour des raisons internes, historiques, notamment celles liées à la jeunesse de sa démocratie, cette gestion au Brésil se fait mal, son efficacité laisse beaucoup à désirer ou s'améliore à un rythme insuffisant, comme montre l'article de Paulo Sergio Pinheiro. Des garanties constitutionnelles, en particulier celles qui concernent les droits civils, souvent peu respectées, ainsi que des conditions précaires de fonctionnement du pouvoir judiciaire et de la police constituent le pendant d'une violence endémique et de violations systématiques des droits humains qui n'ont pas discontinué sous les gouvernements constitutionnels et démocratiques, depuis les années 1990 jusqu'à présent. Cette violence est évoquée dans un contexte plus spécifique, celui d'un Etat fédéré brésilien, Espírito Santo (qui, disons-le au passage, présente - à la fois - des taux d'homicides et un indice de développement humain (IDH) parmi les plus élevés du pays), par le texte de Claudio Zanotelli. Il essaye de comprendre comment se distribuent les homicides dans l'espace social et le territoire urbain, par delà les limites, nous dit-il, d'un dispositif de traitement des données qui reste encore largement insuffisant pour l'ensemble du pays. L'évocation de ces violences se prolonge dans le texte de Sergio Adorno qui montre, pour les villes moyennes de l'intérieur de l'Etat de São Paulo, que l'accroissement de la violence va de pair avec l'accroissement des richesses et garde un lien avec les nouveaux répertoires de consommation des couches moyennes, où les drogues illicites tiennent une place non négligeable ${ }^{4}$.

7 Reste qu'à ces difficultés rencontrées par l'Etat brésilien pour assurer la sécurité des citoyens font écho d'autres, comparables, à l'oeuvre en France comme dans d'autres démocraties européennes. Il est clair, en effet, que l'Etat est aujourd'hui interpellé de façon assez générale dans sa capacité à apporter des réponses efficaces à une violence multiforme qui s'installe dans la vie sociale. Il apparaît affaibli, débordé, son dépérissement est évoqué et certains iraient jusqu'à remettre en cause la célèbre formule de Max Weber lui attribuant le monopole de l'exercice de la violence légitime. Ce débat est au cœur de l'article de Sergio Adorno qui interpelle à ce sujet Michel Wieviorka, lequel à son tour lui répond.

8 La rubrique "Regards sur l'entre-deux », nouvellement créée par la rédaction pour penser l'articulation entre culture et politique, accueille cette fois-ci un dossier sur Glauber Rocha, figure majeure du cinéma brésilien et intellectuel engagé, décédé en 1981 à l'âge de 42 ans. L'article introductif d'Ismail Xavier éclaire le parcours du cinéaste à l'intention d'un - supposé - public de non cinéphiles et met en perspective les textes de Glauber que nous publions ici. Si les cadres qui président à la réflexion de Glauber Rocha sont datés, comme nous le rappelle Ismail Xavier dans son article, sa quête d'une esthétique capable de lier culture et politique a, plus probablement, une valeur atemporelle. Et c'est en cela qu'elle nous intéresse. Antonia Garcia Castro (au nom de la rédaction de Cultures et Conflits) de même que Angelina Peralva et Sergio Adorno (au nom de l'équipe qui a préparé ce numéro) tiennent à exprimer un grand merci - d'abord à Ismail Xavier, qui a accepté de rédiger son article en un temps record, et qui a assuré les contacts indispensables avec l'éditeur brésilien de Glauber Rocha ; à Augusto Massi - qui a mis à notre disposition une photo de couverture appartenant à ses archives personnelles, et à Cosac \& Naify - qui détient les droits sur l'œuvre du cinéaste, sans qui ce dossier n'aurait pas vu le jour; et enfin à Mateus 
Araujo, traducteur et fin connaisseur du travail de Glauber, qui, à Paris, nous a facilité bien des choses.

\section{NOTES}

1.. A l'exception de celui de Claudio Zanotelli, proposé par la rédaction, et du dossier sur Glauber Rocha, élaboré en dehors de ce cadre.

2.. Voir « Síntese de Indicadores Sociais » in Estudos e Pesquisas 12. Informação demográfica e sócioeconômica, IBGE, RJ, 2004.

3.. Données de l'INSERM.

4.. Les données pour l'an 2000 suggèrent que persiste (comme pour les années précédentes) une absence de corrélation statistique directe entre l'IDH des Etats fédérés et les taux d'homicide qu'on y observe. Ainsi, pour les quatre Etats présentant en 2000 les plus forts taux d'homicides observables dans le pays - Pernambouco (54/100 000 h), Rio de Janeiro (51/100 000 h), Espírito Santo (46/100 000 h) et São Paulo $(42 / 100000 \mathrm{~h})$, le rang national dans l'IDH était respectivement le $18^{\text {ème }}$ (sur 27), $5^{\text {ème }}$, $11^{\text {ème }}$ et $3^{\text {ème }}$. Voir IBGE op. cit. et Atlas do Desenvolvimento Humano no Brasil, PNUD.

\section{INDEX}

Mots-clés : démocratie, homicides, violence

Index géographique : Aires géographiques comparées, Amérique du Sud, Brésil, France

\section{AUTEURS}

\section{ANGELINA PERALVA}

Angelina Peralva est sociologue, franco-brésilienne, professeure à l'université de Toulouse II, chercheure au CADIS-CNRS/EHESS, Paris) et associée au CIRUS (CNRS-Université de Toulouse II). Ses recherches portent sur les liens entre violence et démocratie, avec des terrains en France et au Brésil.

\section{SÉRGIO ADORNO}

Sérgio Adorno est professeur au département de sociologie de l'université de São Paulo (Brésil) et directeur du Centre d'Etudes sur la Violence. 